\title{
Treatment of Delayed Rupture of the Left Ventricle After Mitral Valve Replacement
}

\author{
Walter J. Gomes, Malcolm Underwood, Raimondo Ascione, Clinton T. Lloyd, Gianni D. Angelini \\ Bristol, United Kingdom (UK)
}

\begin{abstract}
Rupture of the left ventricle following mitral valve replacement is a catastrophic complication with deadly consequences. We report here the case of a 75-year-old man who underwent elective mitral valve replacement for severe mitral regurgitation. Delayed type 1 rupture of the left ventricle developed 3 hours postoperatively in the intensive care unit. A salvaging maneuver was used, which gained time, allowing reoperation and successful intraventricular repair.
\end{abstract}

Rupture of the left ventricle following mitral valve replacement (MVR) is a catastrophic complication with lethal consequences, which is manifested by massive hemorrhage. The incidence ranges from 0.5 to $1.2 \%$ of all MVR operations ${ }^{1,2}$ with the mortality rate varying between 65 to $100 \%^{3,4}$. This complication is classified according to the time of presentation into early, delayed, and late rupture. Early rupture is defined as taking place in the operative theater following cardiopulmonary bypass discontinuation. Delayed rupture occurs in the early hours after operation, usually in the intensive care unit (ICU), and late is defined as occurring days to years after the operation.

Delayed rupture is the most dreaded scenario because this occurs in the ICU, making it very difficult to control the bleeding and to undertake surgical management. The mortality rate for this condition is considerably high ${ }^{5}$.

Here we report a case where a salvaging maneuver was used in an instance of type 1 left ventricular rupture following MVR occurring in the ICU, which allowed time for reoperation and intraventricular repair.

Bristol Heart Institute, Bristol Royal Infirmary, University of Bristol, Bristol, United Kingdom (UK)

Mailing address: Walter J. Gomes - Cardiovascular Surgery Discipline - EPM Federal University of São Paulo - Rua Botucatu, 740 - São Paulo, SP - Brazil 04023-900 - Email: wjgomes.dcir@epm.br

\section{Case report}

A 75-year-old man underwent to elective MVR for severe mitral regurgitation. He had mild shortness of breath on exertion but was otherwise fit and well. A preoperative transthoracic echocardiogram (TTE) revealed severe mitral regurgitation with a dilated but good left ventricle (end diastolic diameter 6.5 , end systolic diameter $4.4 \mathrm{~cm}$ ). Coronary angiography demonstrated disease-free coronary arteries, and ventriculography showed an ejection fraction of $85 \%$. At operation, the mitral valve was found to be myxomatous with a cleft in the posterior leaflet and a redundant prolapsing anterior leaflet. It was replaced with a $31 \mathrm{~mm}$ Intact bioprosthesis (Medtronic Inc., Minneapolis, USA) with sparing of the posterior leaflet.

The operation was uneventful and the patient was taken to the ICU in stable hemodynamic condition. Three hours later a sudden and profuse drainage of fresh blood was noted from the mediastinal chest drain, along with hemodynamic compromise. Rapid infusion of blood and crystalloid was commenced to restore circulating volume whilst the chest was reopened in the ICU. After gentle medial subluxation of the heart, a 2-cm long tear surrounded by a hematoma was identified in the posterior ventricular wall, alongside the left atrioventricular groove. Blood was jetting from this tear. Using the left hand, the surgeon pressed a small swab against the bleeding point and eventually controlled the hemorrhage. Rapid transfusion of volume allowed improvement in the patient's hemodynamics, and he was taken back to the operative theater.

Cardiopulmonary bypass was reinstituted with bicaval cannulation, the aorta cross-clamped, and warm blood cardioplegia infused intermittently into the aortic root, while the body temperature was maintained normothermic throughout the procedure. The left atrium was reopened and the bioprosthesis removed, after which the tear was seen amidst the hematoma close to the posterior mitral annulus. A glutaraldehyde-preserved bovine pericardium patch was stitched with a continuous 4-0 Prolene suture to the endocardium of the left ventricle, with deep bites in the healthy 
myocardium to completely cover the tear and hematoma from within. A 27-mm Sorin bileaflet mechanical valve was inserted with interrupted 2-0 Ethibond sutures incorporating the bovine pericardium patch. A cellulose patch (Surgicel, Johnson \& Johnson, USA) stuck with cyanoacrylate glue (Glubran, Lucca, Italy) was applied to the epicardium to reinforce the repair and aid hemostasis. The aortic cross-clamp was released, the heart resumed beating in sinus rhythm, and cardiopulmonary bypass was discontinued with minimal inotropic support. An intraaortic balloon pump (IABP) was inserted to offload the left ventricle and reduce tension on the repair suture line. Diffuse bleeding demanded several hours of careful hemostasis. The chest was eventually packed, left open, and the patient returned to the ICU hemodynamically stable. The packs were removed the following day, and the chest closed in the conventional way. The IABP was removed on the second postoperative day and the inotropes weaned. TTE at day 9 demonstrated a good left ventricle (end diastolic diameter 5.4 $\mathrm{cm})$ with hypokinesia of the anterior septum and no ventricular dilatation (fig. 1). The patient was discharged home on day 16. No changes were noted in the cardiac enzymes or ECG.

Two years later, the patient is alive and well. Auscultation demonstrated crisp prosthetic valve clicks with no regurgitant murmur.

\section{Discussion}

Left ventricular rupture after MVR has been classified according to timing (early, delayed, and late) and the site of rupture (type 1,2, and 3). When the tear occurs alongside the left atrioventricular groove (type 1), the outcome is considered extremely grave.

The overall mortality reported in the medical literature ranges from 65 to $100 \%$ of all cases ${ }^{3,4}$, and this high mortality is due to the technical difficulty encountered when repairing the ventricular tear and avoiding damage to the nearby cardiac structures. However, mortality in the delayed form of rupture is far more serious, because it occurs in the ICU. Survival in that former condition is as low as $11 \%$ of patients ${ }^{5}$.

Earlier techniques for correction of this complication involved placement of deep buttressed sutures inside or outside the heart, with the risk of compromising the circumflex artery and causing myocardial infarction. The technique of suturing a pericardial patch to the inside of the left ventricle recovering the tear avoids such a problem but mandates the use of cardiopulmonary bypass, therefore extending the aortic cross-clamp time.

The maneuver used to block the bleeding and hemato-

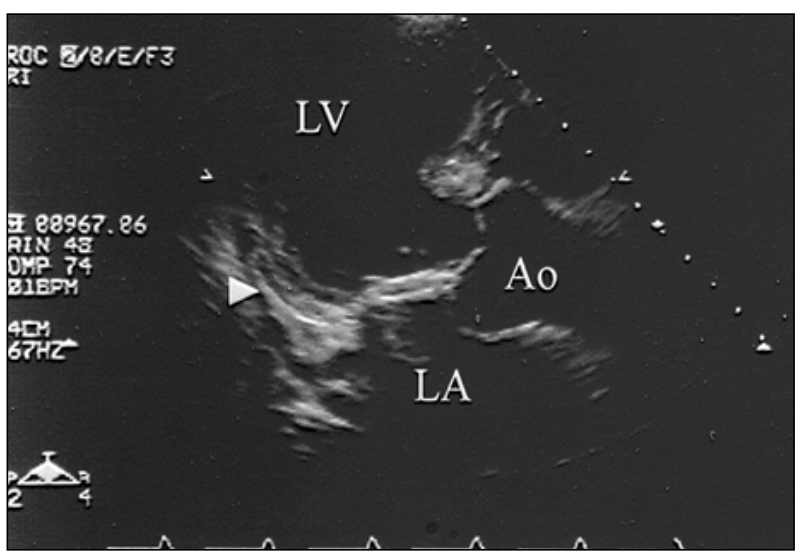

Fig. 1 - Postoperative transthoracic echocardiogram at day 9. Arrow demonstrates the patch repair of the ventricular wall. Ao- aorta; LA- left atrium; LV-left ventricle.

ma expansion was simple and is largely used in the accident and emergency clinic. It proved to be efficient in this scenario and allowed time for the patient to be resuscitated and transferred to the operating theater for commencement of cardiopulmonary bypass. However, care must be taken not to displace the heart too widely as this may extend the tear. The same precaution must be observed after the repair. The operator must avoid lifting the heart to inspect the posterior wall, as this may disrupt the repair with a recurrence of bleeding.

The use of a cellulose patch soaked in glue and applied to the epicardium covering the hematoma has been shown to be a useful and efficient adjuvant technique for controlling the bleeding, because the precise external site of rupture can hardly be identified ${ }^{6}$.

Thorough use of the IABP pump has been advised following the repair aiming to unload the left ventricle and lessen the tension on the injured area, therefore helping to preserve the integrity of the repair ${ }^{7}$, along with prolonged sedation to reduce stress on the repair.

Every effort should be made to prevent this complication and the most critical aspect in type 1 rupture has been reported to be preservation of the posterior mitral valve apparatus ${ }^{8}$. However, as shown in this report, this may not be enough, and further measures should be taken to prevent this catastrophic complication. These include careful stitch placement, minimal traction on the mitral valve annulus during valve excision/insertion, and avoidance of undue manipulation of the heart during de-airing.

In conclusion, we demonstrated that delayed left ventricular rupture after MVR can be managed in the ICU with a simple maneuver, allowing time for hemodynamic restoration and surgical intraventricular repair in the operative theater. 


\section{References}

1. Spencer FC, Galloway AC, Colvin SB. A clinical evaluation of the hypothesis that rupture of the left ventricle following mitral valve replacement can be prevented by preservation of the chordae of the mural leaflet. Ann Surg 1985; 202: 673-80.

2. Jacobowitz IJ, Sabado M, Zisbrod Z, Rose DM, Acinapura J, Cunningham JN Repair of ventricular rupture following mitral valve replacement. J Cardiovasc Surg 1988; 29: 399-402.

3. Cheng LC, Chiu CSW, Lee JWT. Left ventricular rupture after mitral valve replacement. J Cardiovasc Surg 1999; 40: 339-42.

4. Nili M, Salomon J, Halevi A, Schuchman E, Levy MJ. Left ventricular rupture after mitral valve replacement - report of 2 cases and review of the literature. Scand J Thorac Cardiovasc Surg 1981: 15; 235-8.
5. Dark JH, Bain WH. Rupture of posterior wall of the left ventricle after mitral valve replacement. Thorax 1984; 39: 905-11.

6. de la Fuente A, Agudo O, Sanchez R, Fernandez JL, Moriones I. Repair of left ventricular rupture after mitral valve replacement: use of a Teflon patch and glue. Ann Thorac Surg 1999; 67: 1802-3.

7. Yoshida K, Oshima H, Murakami F, et al. Left ventricular free wall rupture following mitral valve replacement. Ann Thorac Cardiovasc Surg 1998; 4 : 336-9.

8. Miller Jr DW, Johnson DD, Ivey TD. Does preservation of the posterior chordae tendineae enhance survival during mitral valve replacement? Ann Thorac Surg $1979 ; 28: 22-7$. 